\title{
Reduction of facial wrinkles by hydrolyzed water- soluble egg membrane associated with reduction of free radical stress and support of matrix production by dermal fibroblasts
}

This article was published in the following Dove Press journal:

Clinical, Cosmetic and Investigational Dermatology

14 October 2016

Number of times this article has been viewed

\section{Gitte S Jensen' \\ Bijal Shah ${ }^{2}$ \\ Robert Holtz ${ }^{3}$ \\ Ashok Patel ${ }^{4}$ \\ Donald C Lo}

'NIS Labs, Klamath Falls, OR, ${ }^{2}$ Department of Neurobiology, Center for Drug Discovery, Duke University Medical Center, Durham, NC, ${ }^{3}$ Biolnnovation Laboratories, Inc., Lakewood, $\mathrm{CO},{ }^{4}$ Centre Manufacturing LLC, Eden Prairie, MN, USA

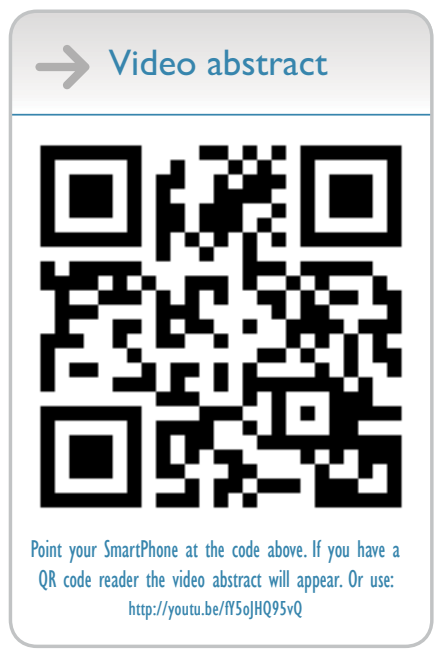

Correspondence: Gitte S Jensen Natural Products Research, NIS Laboratories, I437 Esplanade, Klamath Falls, OR 9760I, USA

$\mathrm{Tel}+|54| 8840112$

$\mathrm{Fax}+\mid 403$ 44| 5236

Email gitte@nislabs.com
Objective: The aim of this study was to evaluate the effects of water-soluble egg membrane (WSEM) on wrinkle reduction in a clinical pilot study and to elucidate specific mechanisms of action using primary human immune and dermal cell-based bioassays.

Methods: To evaluate the effects of topical application of WSEM (8\%) on human skin, an openlabel 8-week study was performed involving 20 healthy females between the age of 45 years and 65 years. High-resolution photography and digital analysis were used to evaluate the wrinkle depth in the facial skin areas beside the eye (crow's feet). WSEM was tested for total antioxidant capacity and effects on the formation of reactive oxygen species by human polymorphonuclear cells. Human keratinocytes (HaCaT cells) were used for quantitative polymerase chain reaction analysis of the antioxidant response element genes Nqo1, Gclm, Gclc, and Hmox1. Evaluation of effects on human primary dermal fibroblasts in vitro included cellular viability and production of the matrix components collagen and elastin.

Results: Topical use of a WSEM-containing facial cream for 8 weeks resulted in a significant reduction of wrinkle depth $(P<0.05)$. WSEM contained antioxidants and reduced the formation of reactive oxygen species by inflammatory cells in vitro. Despite lack of a quantifiable effect on Nrf2, WSEM induced the gene expression of downstream Nqo1, Gclm, Gclc, and Hmox1 in human keratinocytes. Human dermal fibroblasts treated with WSEM produced more collagen and elastin than untreated cells or cells treated with dbcAMP control. The increase in collagen production was statistically significant $(P<0.05)$.

Conclusion: The topical use of WSEM on facial skin significantly reduced the wrinkle depth. The underlying mechanisms of this effect may be related to protection from free radical damage at the cellular level and induction of several antioxidant response elements, combined with stimulation of human dermal fibroblasts to secrete high levels of matrix components.

Keywords: anti-inflammatory, antioxidant response elements, collagen, elastin

\section{Introduction}

Skin is a multifunctional organ, serving important defense functions against external insults to the body, as both a physical barrier toward the external milieu and an immune defense against potentially pathogenic microbial forms. ${ }^{1}$ The skin is subject to both intrinsic (chronological) and extrinsic (environmental) aging, resulting in a loss of functional capacity. ${ }^{2}$ Wrinkle formation is a sign of accelerated aging of skin as an organ and is negatively affected by metabolic dysfunction and loss of glycemic control, ${ }^{3}$ as well as increased blood lipids. Age-related wrinkling in the skin is promoted 
by habitual facial expressions, aging, sun damage, smoking, poor hydration, and various other factors. A substantial harmful influence on the skin aging process includes exposure to ultraviolet (UV) radiation, particularly in combination with pollutants such as polycyclic aromatic polycarbons. ${ }^{4}$ UV radiation speeds up the natural aging process, in part by inducing free radical production in the skin, ${ }^{5}$ and it is the primary cause of accelerated wrinkling. This is in part due to a breakdown of the skin's connective tissue, including collagen and elastin fibers in the deeper layer of the dermis.

Glycosaminoglycans (GAGs) are produced by the body to maintain structural integrity in tissues. Hyaluronic acid is a type of GAG that serves as a natural moisturizer and lubricant between epidermal cells to inhibit the production of matrix metalloproteinases and also promotes collagen synthesis, tissue repair, and hydration. Topical application of GAGs onto skin can help to provide temporary restoration of enzyme balance to slow or prevent matrix breakdown and thereby delay the onset of wrinkle formation.

In addition to the physical barrier function of the skin, the three-dimensional (3D) skin tissue is also an important barrier for invading pathogens. The skin is a highly active immune tissue, ${ }^{6}$ where cells in the skin itself as well as in the microcirculation are active parts of that defense. ${ }^{7}$

Although mammalian skin is a dynamic tissue with the blood and lymphatic circulations providing nutrients and removing waste products, avian eggs are a closed system, where many protective functions are provided by the egg membrane (EM). The natural biological role of avian EM is to act as a scaffold for the formation of the eggshell during development, as well as to provide antimicrobial protection for the growing avian embryo. ${ }^{8,9}$ EM is being investigated as a biological matrix for regeneration in wounds, ${ }^{10,11}$ nerves, ${ }^{12}$ and joint cartilage. EM displays properties including moisture retention and biodegradability and has shown promise for microencapsulation for nutrient delivery. ${ }^{13}$ Proteoglycans in EM have been successfully used in treatments of nonhealing wounds and burns due to the biocompatibility, biodegradability, and similarity to macromolecules found in the human body. ${ }^{14,15}$ Consumption of EM as a nutritional supplement has been associated with significant improvement of physical functioning and range or motion in a population with chronic jointrelated pain. ${ }^{16}$ Of specific interest for the research reported in this article, an earlier study showed that the application of an extract of soluble EM peptides diminished UV-B radiationinduced wrinkle formation in a model of hairless mice, linked to a dose-dependent inhibition of collagenase and also linked to an increase in synthesis of collagen and hyaluronic acid. ${ }^{17}$
The aim of this study was to evaluate the effects of soluble EM peptides when applied topically on human skin and to explore specific mechanisms of action pertaining to skin cell matrix deposition and free radical protection at the cellular level.

\section{Materials and methods Water-soluble egg membrane}

The water-soluble EM (WSEM) product was manufactured by Biova LLC (Johnston, IA, USA) using a patented method combining mechanical and chemical methods of hydrolysis to produce a fine white powder. ${ }^{18}$ For the clinical study, a facial cream was used, containing deionized water, 8\% WSEM (batch \#171014A), olive oil, stearic acid, cetyl palmitate, cetearyl alcohol, cetearyl olivate, and a blend of rosemary oleoresin and vitamin $\mathrm{E}$ acetate as natural preservatives.

\section{Clinical study design}

The evaluation of the effects of topical application of WSEM on human skin was performed using an open-label 8-week study design. The study was conducted at Laboratoire Dermscan, Villeurbanne, France, during the winter months from November 2014 to January 2015. A total of 20 healthy females were enrolled after providing the written informed consent according to the following inclusion criteria: healthy female subjects between the age of 45 years and 65 years with some wrinkles and fine lines in the facial skin beside the eyes (crow's feet). Ethical approval was deemed not necessary by Dermscan. Exclusion criteria were: pregnant, nursing, or planning to get pregnant during the study; having changed, started, or stopped her oral contraceptive or other hormonal treatment within the last 1.5 months; cutaneous pathology on the studied zone (eg, eczema); use of topical or systemic treatment during the previous weeks liable to interfere with the assessment of the efficacy/cutaneous acceptability of the studied product; known allergy to cosmetic or dermopharmaceutical products; excessive exposure to sunlight or UV rays within the previous month; use of antiwrinkles and/or firming products during the last month; previous collagen treatment, face lift, or injections of hyaluronic acid; and having had surgery under general anesthesia within the previous month.

For each subject, the skin area beside the eye was evaluated for wrinkles at baseline, and at 4 weeks and 8 weeks. The imaging was performed directly in vivo, using a 3D system Phaseshift Rapid In vivo Measurement Of Skin (PRIMOS ${ }^{\circledR}$ Lite, GFMesstechnik GmbH, Teltow, Germany). The acquisition software obtained 2D and 3D measurements and determined parameters of the cutaneous contours along 32 radiuses distributed like a star on the zone of interest. An 
automatic system of repositioning allowed the precise reidentification of the zone of measurement. A projection system included a Digital Micromirror Device from Texas Instruments Inc. (Dallas, TX, USA) with $800 \times 600$ micromirrors, use of polarized optics, and a camera with $640 \times 480$ pixels, for a measuring field of $18 \times 13 \mathrm{~mm}^{2}$. The acquisition time of measurement was $17 \mathrm{~ms}$ for one image and $68 \mathrm{~ms}$ for a complete $3 \mathrm{D}$ profile, with a vertical resolution of $1 \mu \mathrm{m}$ and a lateral resolution of $17 \times 17 \mu \mathrm{m}$. Data were based on the average of the 32 profiles from each subject's evaluation at each time point and included the following parameters (Figure 1):

- $\mathrm{Ra}$ : The average roughness (in micrometers): defined as the ratio between the surface integrated around the mean value on the profile length. A decrease in Ra characterizes a smoothing effect.

- Rz: The average contour (in micrometers): average value of all the maxima (between peaks and hollow) on the profile length. A decrease in $\mathrm{Rz}$ characterizes an antiwrinkle effect.

- Rt: The amplitude (in micrometers): average value of the five highest maxima (between peaks and hollow) on the profile length. A decrease in Rt characterizes an antiwrinkle effect.

\section{Total antioxidant capacity assay}

Total antioxidant capacity was evaluated by the Folin-Ciocalteu assay, in which a mixture of phosphomolybdate and

A

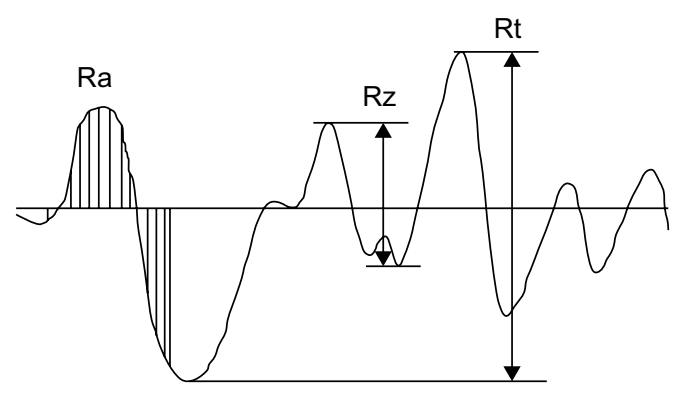

C

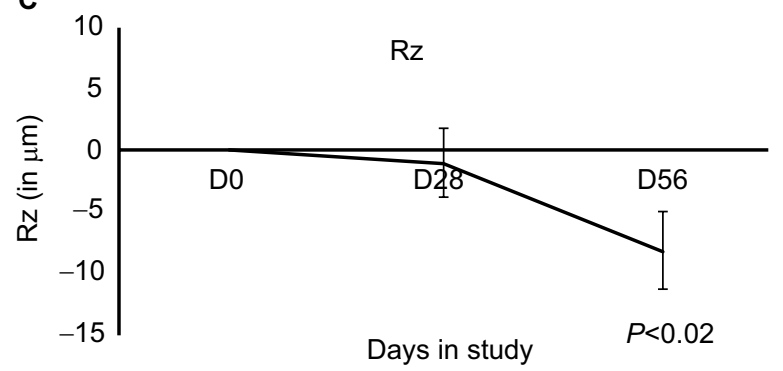

phosphotungstate was used for the colorimetric in vitro assay of phenolic and polyphenolic antioxidants. ${ }^{19}$ The Folin-Ciocalteu's phenol reagent was added to serial dilutions of the test product and incubated for 5 minutes. To start the chemical reaction, sodium carbonate was added, and the reaction was allowed to continue for 30 minutes at $37^{\circ} \mathrm{C}$. Kinetic measurements of the optical absorbance at $765 \mathrm{~nm}$ were read in a colorimetric plate reader (BioTek PowerWave, Winooski, VT, USA).

\section{Reactive oxygen species formation}

Polymorphonuclear (PMN) cells were obtained from healthy human volunteers between the age of 18 years and 65 years, after written informed consent was obtained, as approved by the Sky Lakes Medical Center Institutional Review Board (FWA2603). Isolation of PMN cells was performed as described earlier. ${ }^{20-22}$ The PMN cells were incubated at $37^{\circ} \mathrm{C}$ in $5 \% \mathrm{CO}_{2}$ for 90 minutes, either untreated or treated with serial dilutions of WSEM. The PMN cells were washed twice in phosphate-buffered saline (PBS) to remove any unbound and unabsorbed compounds from the test product. The untreated vs product-treated PMN cells were subsequently loaded with a precursor dye $2^{\prime}, 7^{\prime}$-dichlorofluorescein diacetate and incubated for 1 hour at $37^{\circ} \mathrm{C}$ to allow the precursor dye to be absorbed into the PMN cells. All samples, except for the negative control samples, were then exposed to $167 \mathrm{mM} \mathrm{H}_{2} \mathrm{O}_{2}$ for 45 minutes to induce oxidative stress.
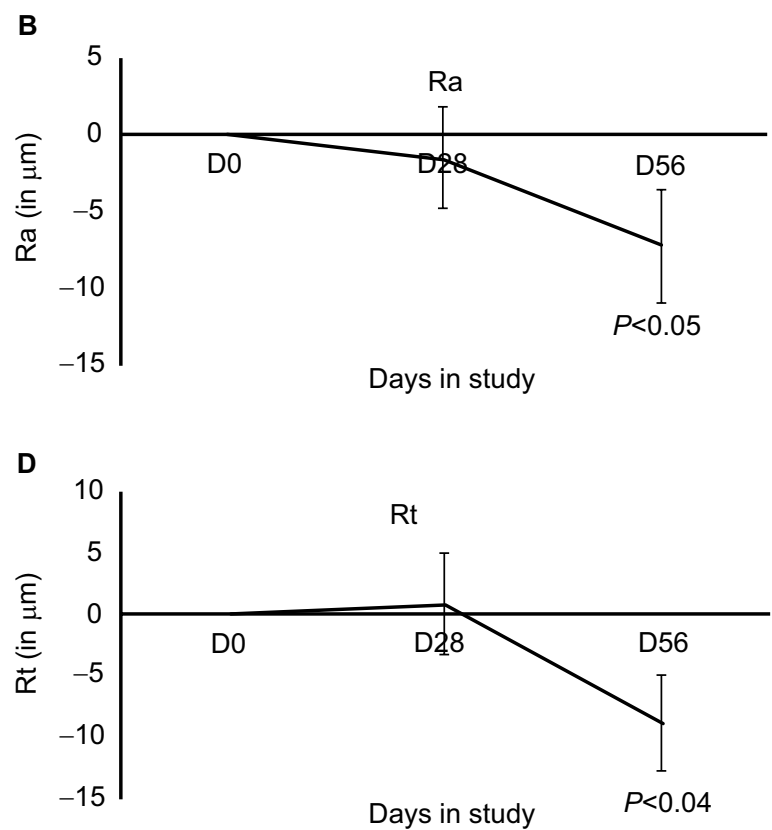

Figure I Wrinkle reduction documented by all the three parameters described in (A) diagram of skin contours and data collection; (B) Ra (average roughness in micrometers); (C) Rz (average contour of all maxima [between peaks and hollows] on the profile length; and [D] Rt [average amplitude in micrometers of the five highest maxima \{between peaks and hollows\}] on the profile length).

Note: The wrinkle reduction seen at day 56 was statistically significant for all the three parameters $(P<0.05)$.

Abbreviation: D, day. 
Samples were washed twice in PBS to remove the peroxide, transferred to ice-cold RPMI 1640 medium, and stored on ice in the dark. The $2^{\prime}, 7^{\prime}$-dichlorofluorescein diacetate fluorescence intensity was immediately measured by flow cytometry (Attune; Thermo Fisher Scientific, Waltham, MA, USA). Data were collected in triplicate for controls and for each dose of the extracts of test products. The mean fluorescence intensity of PMN cells was compared between untreated, $\mathrm{H}_{2} \mathrm{O}_{2}$-treated, and product-pretreated cells. An increase in fluorescence intensity would indicate stimulation of the innate immune response by the PMN cells, and a reduction in mean fluorescence intensity in samples pretreated with WSEM prior to challenge with $\mathrm{H}_{2} \mathrm{O}_{2}$ would indicate that WSEM was able to reduce the formation of reactive oxygen species (ROS) in PMN cells under conditions of oxidative stress.

\section{Transcriptional induction of endogenous antioxidant response element antioxidant genes}

Transcriptional induction of endogenous antioxidant response element (ARE) antioxidant genes was evaluated at Duke University Medical Center using HaCaT cells, a spontaneously transformed human epithelial cell line from adult skin, which maintains full epidermal differentiation capacity. ${ }^{23}$ This human keratinocyte line retains the ability to form epithelial structures upon transplantation into mice and is a widely used in vitro model for keratinocyte differentiation and function. HaCaT cells were procured from AddexBio (San Diego, CA, USA).

Levels of expression of the candidate target ARE antioxidant genes Nqo1, Gclm, Gclc, and Hmoxl were measured by realtime quantitative polymerase chain reaction (qPCR). For these measurements, 350,000 HaCaT cells were plated per well in sixwell plates. Compounds were added 18-20 hours after plating; cells were harvested for qPCR analysis 6 hours after treatment. Total RNA was isolated from cell lysates using Absolutely RNA Mini-Prep Kits (Agilent Technologies, Santa Clara, CA, USA). cDNA was generated using Oligo dT primers and SuperScript II Reverse Transcriptase (Thermo Fisher Scientific). Resulting cDNA was used for qPCR of gene transcripts using Power SYBR Green PCR Mastermix (Thermo Fisher Scientific) and primer sequences as published earlier. ${ }^{24}$ Each biological sample was measured in triplicate on a ViiA 7 real-time PCR instrument (Thermo Fisher Scientific); fold expression was calculated after normalization to corresponding control $\beta$-actin levels with the dimethyl-sulfoxide-only control condition set to a value of 1 .

\section{Human dermal fibroblast cultures}

Adult human dermal fibroblasts were obtained from Thermo Fisher Scientific, seeded into the individual wells of a 24-well plate in $0.5 \mathrm{~mL}$ of fibroblast growth media (Dulbecco's Modified Eagle's Medium [DMEM] supplemented with 2\% fetal bovine serum [FBS], $1 \mathrm{ng} / \mathrm{mL}$ basic fibroblast growth factor, and $10 \mu \mathrm{g} / \mathrm{mL}$ insulin, where all reagents were obtained from Sigma-Aldrich Co., St Louis, MO, USA), and incubated overnight at $37^{\circ} \mathrm{C}$ in $5 \% \mathrm{CO}_{2}$. On the following day, the medium was removed by aspiration to eliminate any nonadherent cells and replaced with $0.5 \mathrm{~mL}$ of fresh Fibroblast Growth Medium. The cells were grown until confluent, with a media change every 48-72 hours. Upon reaching confluency, the cells were treated for 24 hours with DMEM supplemented with $1.5 \%$ FBS to wash out any effects from the growth factors included in the normal culture media. After this 24-hour washout period, the cells were treated with WSEM at the specified concentrations dissolved in Fibroblast Growth Medium with $1.5 \%$ FBS. Transforming growth factor- $\beta$ $(50 \mathrm{ng} / \mathrm{mL})$ was used as a positive control for collagen and elastin synthesis, and dibutyl cAMP $(0.1 \mathrm{mM})$ was used as a positive control for inducing hyaluronic acid production. Untreated cells (negative controls) received DMEM with $1.5 \%$ FBS. The cells were incubated for 48 hours, and at the end of the incubation period, the culture supernatants were collected and either stored frozen $\left(-75^{\circ} \mathrm{C}\right)$ or assayed immediately. Each dose of WSEM was tested in triplicate.

\section{MTT assay}

To test the effects of test products on cell proliferation, the MTT assay was used, where the tetrazolium dye MTT is reduced to insoluble purple formazan, which has a purple color. After the 48-hour incubation, the cell culture medium was removed (as mentioned earlier) and the fibroblasts were washed twice with PBS to remove any remaining test materials. After the final wash, $500 \mu \mathrm{L}$ of DMEM supplemented with $0.5 \mathrm{mg} / \mathrm{mL}$ MTT was added to each well and the cells were incubated for 1 hour at $37^{\circ} \mathrm{C} \pm 2^{\circ} \mathrm{C}$ and $5 \% \pm 1 \% \mathrm{CO}_{2}$. After the incubation, the DMEM/MTT solution was removed and the cells were washed once with PBS, after which $0.5 \mathrm{~mL}$ of isopropyl alcohol was added to the well to extract the purple formazan crystals. Samples were transferred to a 96-well plate and the plate was read at $540 \mathrm{~nm}$, with isopropyl alcohol as a negative control. The mean absorbance value for the negative control cells was calculated and used to represent $100 \%$ cell viability. Individual absorbance values from the cell cultures treated with WSEM vs positive and negative controls were then divided by the mean value of 
the negative control cells and expressed as a percentage to document the change in cell viability caused by each treatment.

\section{Procollagen assay}

Standards of type I-C peptide were prepared ranging from $0 \mathrm{ng} / \mathrm{mL}$ to $640 \mathrm{ng} / \mathrm{mL}$. An enzyme-linked immunosorbent assay (ELISA) microplate was prepared by the addition of $100 \mu \mathrm{L}$ of peroxidase-labeled anti-procollagen type I-C peptide antibody per well. Then, $20 \mu \mathrm{L}$ volume of either standard or sample (collected tissue culture media) was added to appropriate wells and allowed to incubate for $3 \pm 0.25$ hours at $37^{\circ} \mathrm{C}$. After the incubation, the suspension was removed by aspiration and washed three times with $400 \mu \mathrm{L}$ of wash buffer. After the last wash, $100 \mu \mathrm{L}$ of peroxidase substrate solution (hydrogen peroxide + tetramethylbenzidine as a chromogen) was added to each well and the plate was incubated for $15 \pm 5$ minutes at room temperature. After the incubation, $100 \mu \mathrm{L}$ of stop solution ( $1 \mathrm{~N}$ sulfuric acid) was added to each well and the plate was read using a microplate reader (Packard SpectraCount, Meriden, CT, USA) at $450 \mathrm{~nm}$. To quantify the amount of type I-C peptide present, a standard curve was generated using the known concentrations of type I-C peptide. Absorbance values for the test material and untreated samples were used to estimate the amount of each substance present in each sample.

\section{Elastin competitive ELISA}

Soluble $\alpha$-elastin was prepared for the assay by dissolving in $0.1 \mathrm{M}$ sodium carbonate $(\mathrm{pH} 9.0)$ at a concentration of $1.25 \mu \mathrm{g} / \mathrm{mL}$. Then, $150 \mu \mathrm{L}$ of this solution was applied to the wells of a 96-well Maxisorp plate (Nunc, Roskilde, Denmark), and the plate was incubated overnight at $4^{\circ} \mathrm{C}$. On the following day, the wells were saturated with PBS containing $0.25 \%$ bovine serum albumin (BSA) and $0.05 \%$ Tween 20. The plate was then incubated with this blocking solution for 1 hour at $37^{\circ} \mathrm{C}$ and then washed two times with PBS containing $0.05 \%$ Tween 20 . A set of $\alpha$-elastin standards was generated ranging from $0 \mathrm{ng} / \mathrm{mL}$ to $100 \mathrm{ng} / \mathrm{mL}$. Then, $180 \mu \mathrm{L}$ of either standard or sample was transferred to a $650 \mu \mathrm{L}$ microcentrifuge tube. An anti-elastin antibody solution was prepared (1:500 in PBS containing 0.25\% BSA and $0.05 \%$ Tween 20 ), and a volume of $20 \mu \mathrm{L}$ was added to each tube, after which the tubes were incubated overnight at $4^{\circ} \mathrm{C} \pm 2^{\circ} \mathrm{C}$. The next morning, $150 \mu \mathrm{L}$ was transferred from each tube to the 96-well elastin ELISA plate, and the plate was incubated for 1 hour at room temperature. The plate was then washed three times with PBS containing 0.05\%
Tween 20 , and $200 \mu \mathrm{L}$ of peroxidase-linked secondary antibody (diluted in PBS [1:2,500] containing 0.25\% BSA and $0.05 \%$ Tween 20 ) was added, and the plate was incubated at room temperature for 1 hour. After three washes, $200 \mu \mathrm{L}$ of substrate solution was added. The plate was incubated for 10-30 minutes at room temperature in the dark, after which the plate was read at $460 \mathrm{~nm}$ using a plate reader (Packard SpectraCount).

\section{Statistical analysis}

Average, standard deviation, and standard error of the mean were calculated using Microsoft Excel. Statistical analysis of in vitro data was performed using the twotailed, dependent $t$-test. Statistical analysis of the changes over time for the clinical data from the open-label study was performed as "within-subject" analysis using the twotailed, paired $t$-test. Statistical significance was indicated if $P<0.05$.

\section{Results \\ Clinical 8-week study on wrinkle reduction}

Wrinkle reduction was seen for all three parameters: roughness, contour, and wrinkle depth (Figure 1). Timing to wrinkle reduction varied, and some study participants showed robust wrinkle reduction already at 4 weeks; however, this change was not statistically significant within the entire study population. The wrinkle reduction reached statistical significance at 8 weeks. An example of the imaging, based on which the $\mathrm{Ra}, \mathrm{Rz}$, and Rt were calculated, is shown as the individual baseline and day-56 data (Figure 2).

\section{Reduction of free radical stress}

The WSEM contained antioxidants, which was documented in the Folin-Ciocalteu assay (Figure 3A). In addition to its direct antioxidant capacity, WSEM treatment of human primary PMN cells under conditions of oxidative stress resulted in reduced formation of ROS, indicating anti-inflammatory properties (Figure 3B).

\section{Antioxidant gene responses}

WSEM was tested in cell-based assays for Nrf2-mediated activation of innate antioxidant gene responses. Despite the lack of a quantifiable effect on Nrf2 activation in a less sensitive GFP reporter model, WSEM showed moderate but consistent induction of multiple ARE target genes in the HaCaT human keratinocyte cell lines using qPCR analysis (Figure 4). Earlier studies had identified a representative set 


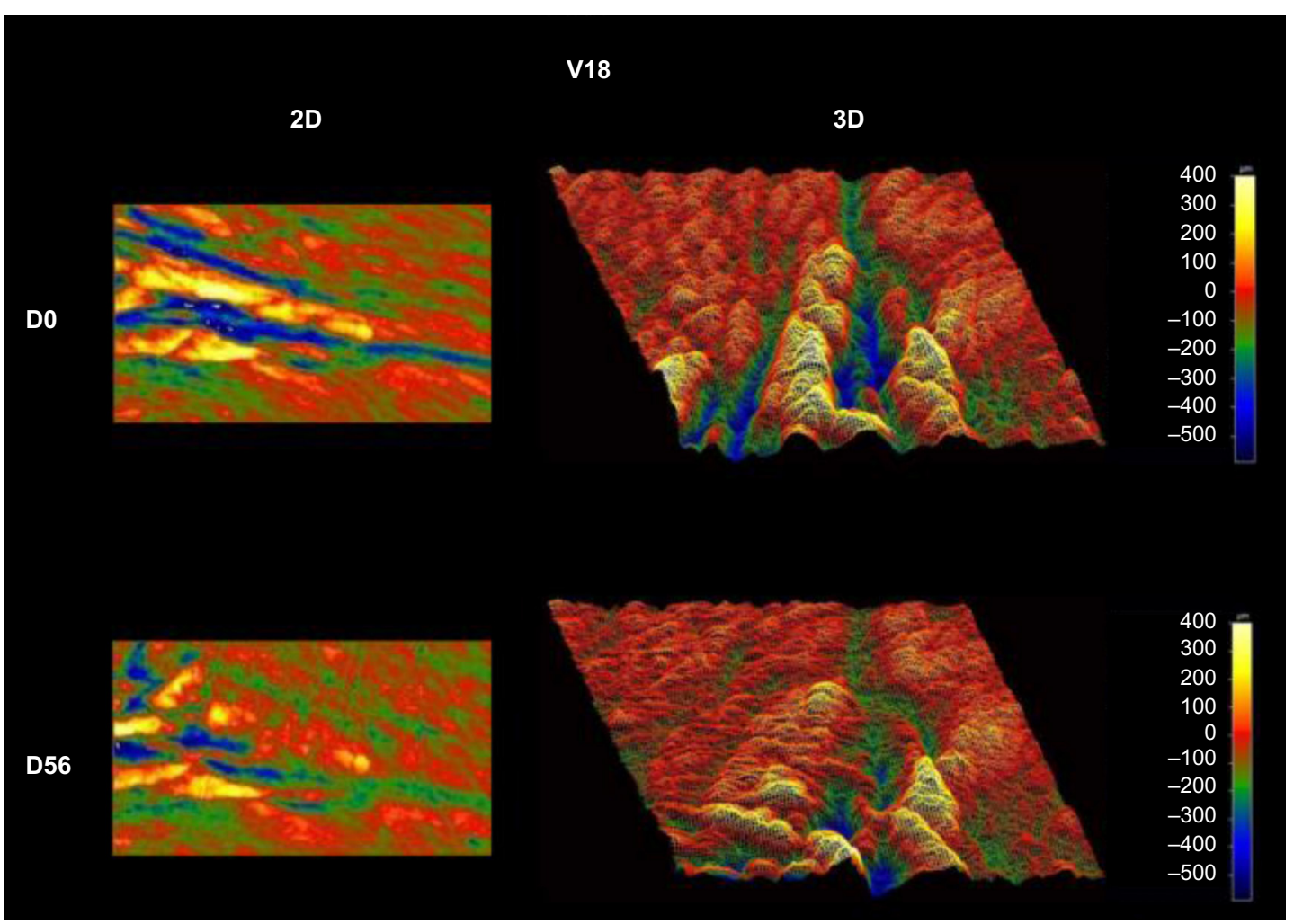

Figure 2 Example of wrinkle reduction over the 56-day clinical study.

Notes: The images to the left are 2D, and the images to the right are 3D. Wrinkle depth is illustrated as increasing scale of blue colors, whereas increasing yellow color reflects the magnitude of the wrinkle crest.

Abbreviations: D, day; 2D, two dimensional; 3D, three dimensional.
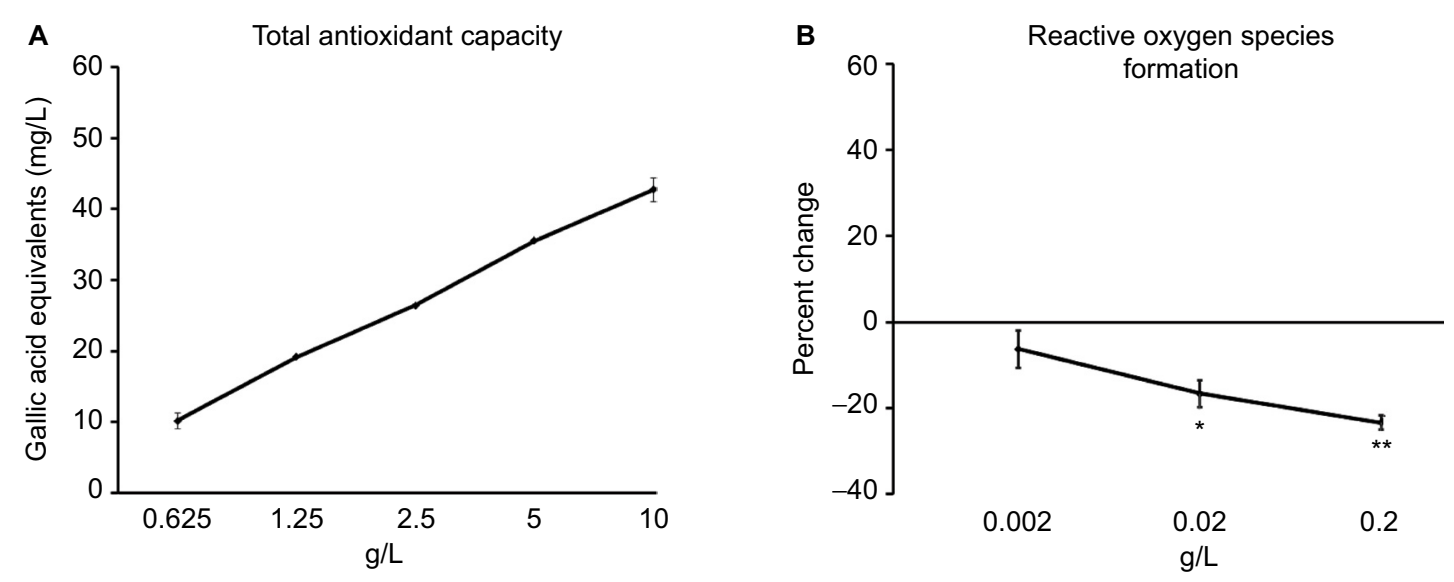

Figure 3 Reduction of free radical stress by WSEM.

Notes: (A) Total antioxidant capacity was documented in the Folin-Ciocalteu assay, where a broad dose range of WSEM was tested, and the average \pm standard deviation of each duplicate data set is shown. (B) The formation of ROS by human PMN cells. The anti-inflammatory inhibition of the formation of ROS was highly significant at the $0.2 \mathrm{~g} / \mathrm{L}$ dose $(* * P<0.00 \mathrm{I})$, and remained significant at the $0.02 \mathrm{~g} / \mathrm{L}$ dose $(* P<0.03)$.

Abbreviations: PMN, polymorphonuclear; ROS, reactive oxygen species; WSEM, water-soluble egg membrane.

of four ARE target genes that are strongly induced by Nrf2 activation in $\mathrm{HaCaT}$ cells. ${ }^{24}$ These four ARE target genes were: Nqol; Gclm; Gclc; and Hmoxl. The positive control compounds used were dimethylfumarate and the CDDO triterpenoid bardoxolone. As expected, dimethylfumarate and bardoxolone strongly induced the expression levels of
Nqo 1, Gclm, Gclc, and Hmox 1. Interestingly, WSEM was also observed to induce strong increases in mRNA levels of Nqo1, Gclm, and Gclc, and to a lesser but significant extent mRNA levels of Hmoxl (Figure 4). These data suggest that WSEM contains components that could be capable of inducing ARE gene-mediated antioxidant responses. 

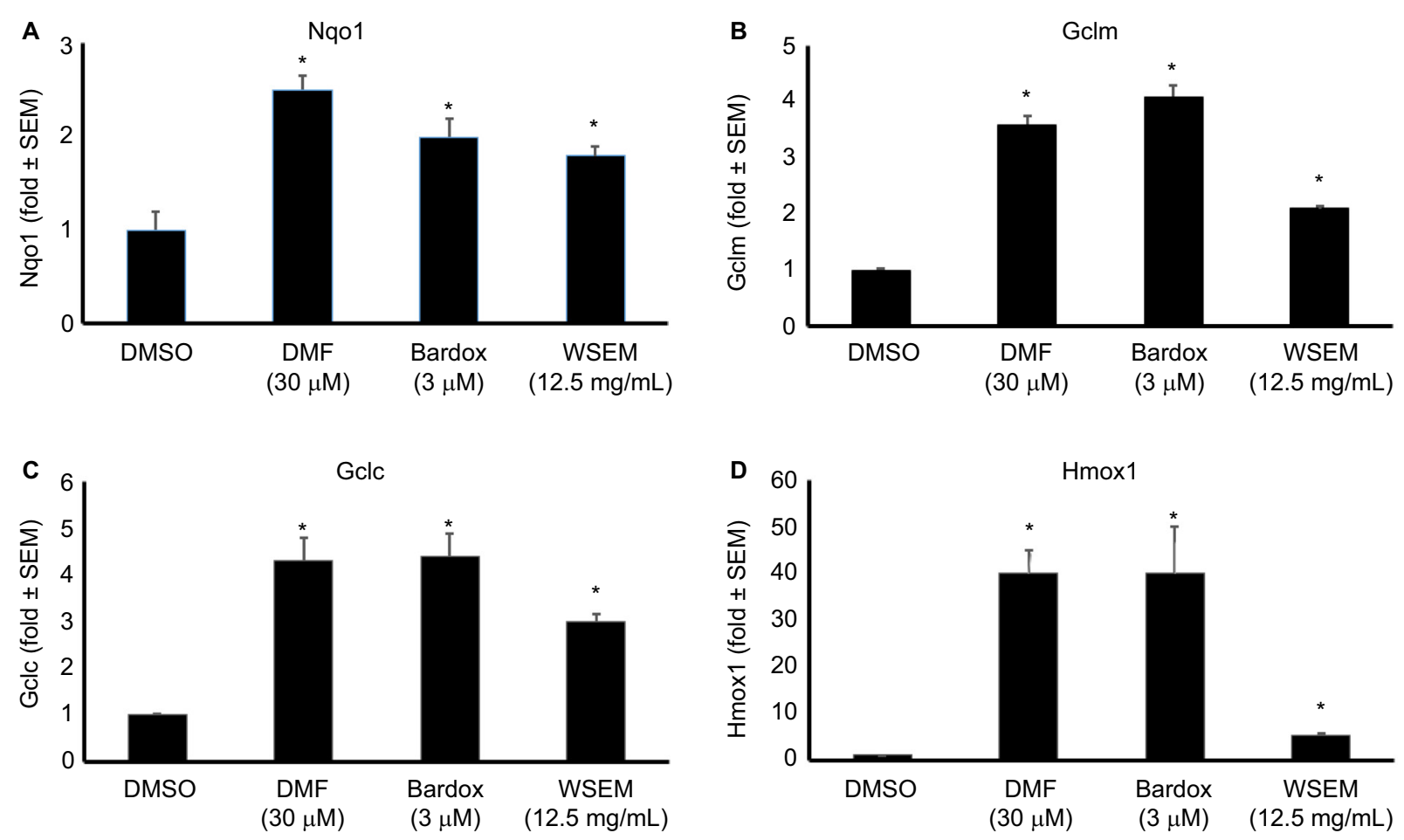

Figure 4 mRNA levels of the ARE genes Nqol (A), Gclm (B), Gclc (C), and Hmox I (D) were quantified using qPCR analysis.

Notes: For this testing, $\mathrm{HaCaT}$ cells were plated at 350,000 cells/well in six-well plate formats. Compounds were added I8-20 hours after plating; cells were harvested for qPCR analysis 6 hours after treatment. Data are expressed as fold change relative to the DMSO-only control condition. WSEM significantly increased mRNA levels of all the four ARE antioxidant response genes evaluated $(P<0.05)$. All treatments induced statistically significant differences compared to the DMSO-only control $(* P<0.05)$. Data are representative of two independent experiments.

Abbreviations: ARE, antioxidant response element; Bardox, bardoxolone; DMF, dimethylfumarate; DMSO, dimethyl sulfoxide; mRNA, messenger RNA; qPCR, quantitative polymerase chain reaction; WSEM, water-soluble egg membrane.

\section{Dermal fibroblast proliferation and matrix production}

The effects of WSEM on human dermal fibroblasts were tested in vitro, where WSEM showed a mild but significant increase in the proliferation of dermal fibroblasts (Figure 5A). WSEM-treated dermal fibroblasts showed a mild but insignificant increase in elastin production (Figure 5B) and a significant increase in collagen production (Figure 5C).

\section{Discussion}

Preservation of healthy skin tissue is of importance for physical protection against environmental pollution and radiation (UV and other), as well as for maintaining and supporting an optimal immune defense activity, such as protection from bacterial and viral infectious agents and maintaining a beneficial cutaneous microbiome, ${ }^{25}$ as well as elimination of malignantly transformed cells after excessive sun exposure via recruitment of immune cells from the microcirculation. ${ }^{26}$ The current study was conducted to evaluate whether topical application of WSEM peptides would be associated with improved skin health, as well as to address specific potential mechanisms of action pertaining to the health of skin tissue.

A significant reduction of facial wrinkles was seen after 8 weeks of daily application of WSEM in the open-label clinical pilot study. These effects on wrinkle reduction may be a combined effect of the content of elastin in WSEM, the stimulation of matrix production in dermal cells in the skin treated with WSEM, and other regulating effects on the reduction of free radical stress.

The effects of WSEM on primary human dermal fibroblasts in culture may help explain mechanisms behind the restorative effects seen in the clinical study. The increase in collagen and elastin production may be a direct causative effect for the smoothing effects seen on facial wrinkles in the clinical study. This moderate increase in collagen and elastin production is interesting but warrants further work using a more direct methodology. Further testing should include visualization of newly deposited collagen and elastin in confluent cultures of dermal fibroblasts with anti-collagen and anti-elastin antibodies, as a more definitive morphometric assessment of fully assembled components of the dermal matrix in fibroblast cultures.

It has been shown that dermal fibroblasts isolated from older donors were more sensitive to oxidative stress than fibroblasts from younger donors, ${ }^{27}$ and it seems plausible that WSEM may offer a natural method to reduce oxidative stress in skin tissue, thus contributing to a protective antiaging effect. The protection from free radical damage by WSEM included i) direct antioxidant capacity, ii) reduction of ROS formation 

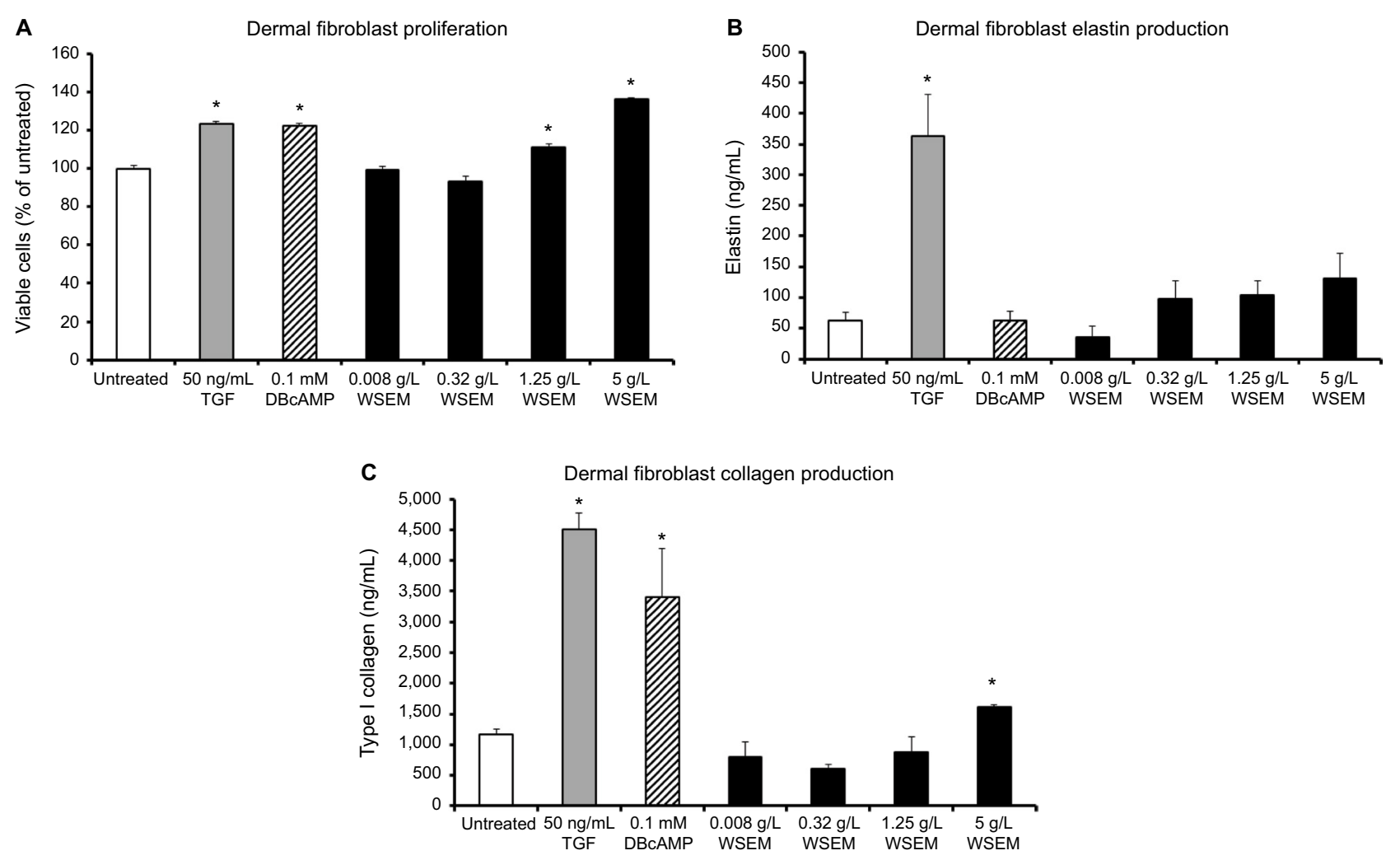

Figure 5 The effects of WSEM on human dermal fibroblasts are shown as the average \pm standard deviation of triplicate cultures.

Notes: WSEM showed a mild but significant increase in the proliferation of dermal fibroblasts (A). In addition, WSEM-treated dermal fibroblasts showed an increase in the production of elastin (B) and collagen (C). The increases in elastin and collagen levels in the dermal fibroblast cultures were not due to the content of those compounds in WSEM, since the $5 \mathrm{~g} / \mathrm{L}$ dose of WSEM showed similar levels as the negative control (medium alone) at or below $6 \mathrm{ng} / \mathrm{mL}(\mathrm{data} n$ not shown). Statistical significance $(P<0.05)$ is calculated compared to untreated cell cultures, and where significance was reached indicated by *.

Abbreviations: TGF, transforming growth factor;WSEM, water-soluble egg membrane.

by inflammatory cells, as well as iii) induction of ARE genes in keratinocytes. The $80 \mathrm{~g} / \mathrm{L}$ dose of WSEM used in the clinical study was higher than the $1 \mathrm{~g} / \mathrm{L}$ and lower doses evoking responses in the in vitro bioassay. The epidermal penetration and absorption of active compounds in WSEM in the dermis and microcirculation are unknown. We suggest that if only a proportion of the bioactive peptides in WSEM reach the deeper layers in the dermis, as well as the microvascular circulation, then the in vitro data may indeed help predict events in the living skin during the topical use of WSEM. The protective effects of Nrf2 inducers on human skin, both at a preventive and reconstructive level, are well documented. ${ }^{28,29}$ Therefore, direct effects of WSEM on the redox-sensitive Nrf2-mediated transcriptional induction of four ARE target genes in human HaCaT keratinocytes point to additional mechanisms of action, whereby WSEM may support skin health and restoration, especially under conditions of oxidative stress.

\section{Conclusion}

The results reported in this study suggest that the complex peptide matrix in hydrolyzed EM holds promise in the support of skin health, especially in protection from free radicalinduced damage to the tissue. The effects were seen on three different cell types that are interconnected in the process of age-related wrinkle formation: dermal fibroblasts, dermal keratinocytes, and PMN cells from the blood circulation. Paracrine communication between these three cell types includes the formation of ROS and cytokine production including interleukin- $6^{30}$ and contributes to wrinkle formation through the upregulation of fibroblast elastases, resulting in the loss of elastic properties of the skin. ${ }^{31}$ Further work is needed to establish the effects of WSEM in wound healing and repair, as well as immune protection in common inflammatory skin conditions, including acne. Further studies should include a placebo-controlled study for the observation of wrinkle reduction, including subjects of both sexes. In addition, the data on the reduction of oxidative stress warrant studies on preventative and restorative effects on sunburn-induced skin damage.

\section{Acknowledgments}

The clinical study was conducted at Laboratoire Dermscan, Villeurbanne, France, coordinated by Cambridge Commodities Ltd., Cambridgeshire, UK. The evaluation of antioxidant protection was conducted at NIS Laboratories, an independent contract research laboratory specializing in natural products research. The testing of dermal fibroblasts was conducted at 
BioInnovation Laboratories, Inc., Lakewood, CO, USA. The evaluation of AREs was conducted at the Center for Drug Discovery and Department of Neurobiology, Duke University, Durham, NC, USA, sponsored by Nerium Biotech Inc. The clinical study and the laboratory testing at NIS Laboratories and BioInnovation Laboratories Inc. was sponsored by Biova LLC, the manufacturer and distributor of hydrolyzed WSEM.

\section{Disclosure}

The authors have no financial interest in the subject matter. The clinical study was sponsored by Biova LLC, the manufacturer and distributor of hydrolyzed WSEM. The testing for antioxidant response elements was sponsored by Nerium Biotech Inc. The laboratory testing for antioxidant properties and effects on dermal fibroblasts was sponsored by Biova LLC. The authors report no other conflicts of interest in this work.

\section{References}

1. Pfliger JF. The epidermal skin barrier: implications for the wound care practitioner, Part I. Adv Skin Wound Care. 2004;17(8):417-425.

2. Newton VL, Mcconnell JC, Hibbert SA, Graham HK, Watson RE. Skin aging: molecular pathology, dermal remodelling and the imaging revolution. G Ital Dermatol Venereol. 2015;150(6):665-674.

3. Danby FW. Nutrition and aging skin: sugar and glycation. Clin Dermatol. 2010;28(4):409-411.

4. Borska L, Andrys C, Krejsek J, et al. Influence of dermal exposure to ultraviolet radiation and coal tar (polycyclic aromatic hydrocarbons) on the skin aging process. J Dermatol Sci. 2016;81(3):192-202.

5. Wölfle U, Seelinger G, Bauer G, Meinke MC, Lademann J, Schempp CM. Reactive molecule species and antioxidative mechanisms in normal skin and skin aging. Skin Pharmacol Physiol. 2014;27(6):316-332.

6. Dreno B, Gollnick HP, Kang S, et al; Global Alliance to Improve Outcomes in Acne. Understanding innate immunity and inflammation in acne: implications for management. $J$ Eur Acad Dermatol Venereol. 2015;29(suppl 4):3-11.

7. Said A, Weindl G. Regulation of dendritic cell function in inflammation. J Immunol Res. 2015;2015:743169.

8. Ahlborn G, Sheldon BW. Identifying the components in eggshell membrane responsible for reducing the heat resistance of bacterial pathogens. J Food Prot. 2006;69(4):729-738.

9. Abdel Mageed AM, Isobe N, Yoshimura Y. Immunolocalization of avian beta-defensins in the hen oviduct and their changes in the uterus during egg formation. Reproduction. 2009;138(6):971-978.

10. Yang JY, Chuang SS, Yang WG, Tsay PK. Egg membrane as a new biological dressing in split-thickness skin graft donor sites: a preliminary clinical evaluation. Chang Gung Med J. 2003;26(3):153-159.

11. Maeda K, Sasaki Y. An experience of hen-egg membrane as a biological dressing. Burns. 1981;8(5):313-316.

12. Farjah GH, Heshmatian B, Karimipour M, Saben A. Using eggshell membrane as nerve guide channels in peripheral nerve regeneration. Iran J Basic Med Sci. 2013;16(8):901-905.

13. Chai Z, Li Y, Liu F, et al. Outer eggshell membrane as delivery vehicle for polysaccharide/protein microcapsules incorporated with vitamin $\mathrm{E}$. J Agric Food Chem. 2013;61(3):589-595.
14. Mogosanu GD, Grumezescu AM. Natural and synthetic polymers for wounds and burns dressing. Int J Pharm. 2014;463(2):127-136.

15. Ohto-Fujita E, Konno T, Shimizu M, et al. Hydrolyzed eggshell membrane immobilized on phosphorylcholine polymer supplies extracellular matrix environment for human dermal fibroblasts. Cell Tissue Res. 2011;345(1):177-190.

16. Jensen GS, Lenninger MR, Beaman JL, Taylor R, Benson KF. Support of joint function, range of motion, and physical activity levels by consumption of a water-soluble egg membrane hydrolyzate. J Med Food. 2015;18(9):1042-1048.

17. Yoo JH, Kim JK, Yang HJ, Park KM. Effects of egg shell membrane hydrolysates on UVB-radiation-induced Wrinkle formation in SKH-1 hairless mice. Korean J Food Sci Anim Resour. 2015;35(1):58-70.

18. Strohbehn RE, Etzel LR, Figgins J. Method of separating components of technical eggs, edible eggs, yolk and whites and products therefrom. US Patent No US 8,425,943 B2, 2013 Apr.

19. Singleton VL, Orthofer R, Lamuela-Raventós RM. Analysis of total phenols and other oxidation substrates and antioxidants by means of Folin-Ciocalteu reagent. Meth Enzymol. 1999;299:152-178.

20. Honzel D, Carter SG, Redman KA, Schauss AG, Endres JR, Jensen GS. Comparison of chemical and cell-based antioxidant methods for evaluation of foods and natural products: generating multifaceted data by parallel testing using erythrocytes and polymorphonuclear cells. $J$ Agric Food Chem. 2008;56(18):8319-8325.

21. Jensen GS, Patterson KM, Yoon I. Yeast culture has anti-inflammatory effects and specifically activates NK cells. Comp Immunol Microbiol Infect Dis. 2008;31(6):487-500.

22. Jensen GS, Benson KF, Carter SG, Endres JR. GanedenBC30 cell wall and metabolites: anti-inflammatory and immune modulating effects in vitro. BMC Immunol. 2010;24:11-15.

23. Boukamp P, Petrussevska RT, Breitkreutz D, Hornung J, Markham A, Fusenig NE. Normal keratinization in a spontaneously immortalized aneuploid human keratinocyte cell line. J Cell Biol. 1988; 106:761-771.

24. Zhao R, Hou Y, Zhang Q, et al. Cross-regulations among NRFs and KEAP1 and effects of their silencing on arsenic-induced antioxidant response and cytotoxicity in human keratinocytes. Environ Health Perspect. 2012;120:583-589.

25. Holmes CJ, Plichta JK, Gamelli RL, Radek KA. Dynamic role of host stress responses in modulating the cutaneous microbiome: implications for wound healing and infection. Adv Wound Care (New Rochelle). 2015;4(1):24-37.

26. Jókai H, Marschalkó M, Csomor J, et al. Tissue-specific homing of immune cells in malignant skin tumors. Pathol Oncol Res. 2012;18(4):749-759.

27. Brun C, Jean-Louis F, Oddos T, Bagot M, Bensussan A, Michel L. Phenotypic and functional changes in dermal primary fibroblasts isolated from intrinsically aged human skin. Exp Dermatol. 2016;25(2):113-119.

28. Tao S, Justiniano R, Zhang DD, Wondraka GT. The Nrf2-inducers tanshinone I and dihydrotanshinone protect human skin cells and reconstructed human skin against solar simulated UV. Redox Biol. 2013;1(1):532-541.

29. Saw CL, Huang MT, Liu Y, Khor TO, Conney AH, Kong AN. Impact of Nrf2 on UVB-induced skin inflammation/photoprotection and photoprotective effect of sulforaphane. Mol Carcinog. 2011;50(6): 479-486.

30. Inui M, Ooe M, Fujii K, Matsunaka H, Yoshida M, Ichihashi M. Mechanisms of inhibitory effects of CoQ10 on UVB-induced wrinkle formation in vitro and in vivo. Biofactors. 2008;32(1-4):237-243.

31. Imokawa G. Mechanism of UVB-induced wrinkling of the skin: paracrine cytokine linkage between keratinocytes and fibroblasts leading to the stimulation of elastase. J Invest Derm. 2009;14(1):36-43. 


\section{Publish your work in this journal}

Clinical, Cosmetic and Investigational Dermatology is an international, peer-reviewed, open access, online journal that focuses on the latest clinical and experimental research in all aspects of skin disease and cosmetic interventions. This journal is included on PubMed. The manuscript management system is completely online and includes a very quick and fair peer-review system, which is all easy to use. Visit http://www.dovepress.com/testimonials.php to read real quotes from published authors 